\title{
Classification of the electronic correlation strength in the iron pnictides: The case of the parent compound $\mathrm{BaFe}_{2} \mathrm{As}_{2}$
}

\author{
S. L. Skornyakov, ${ }^{1}$ A. V. Efremov, ${ }^{1}$ N. A. Skorikov, ${ }^{1}$ M. A. Korotin, ${ }^{1}$ Yu. A. Izyumov,,${ }^{1}$ V. I. Anisimov, ${ }^{1}$ A. V. Kozhevnikov, ${ }^{2}$ \\ and D. Vollhardt ${ }^{3}$ \\ ${ }^{1}$ Institute of Metal Physics, Russian Academy of Sciences, 620041 Yekaterinburg GSP-170, Russia \\ ${ }^{2}$ Joint Institute for Computational Sciences, Oak Ridge National Laboratory, P.O. Box 2008, Oak Ridge, Tennessee 37831-6173, USA \\ ${ }^{3}$ Theoretical Physics III, Center for Electronic Correlations and Magnetism, Institute for Physics, University of Augsburg, \\ Augsburg 86135, Germany \\ (Received 17 June 2009; revised manuscript received 23 July 2009; published 3 September 2009)
}

\begin{abstract}
Electronic correlations in the Fe-pnictide $\mathrm{BaFe}_{2} \mathrm{As}_{2}$ are explored within LDA+DMFT, the combination of density functional theory with dynamical mean-field theory. While the correlated band structure is substantially renormalized there is only little transfer of spectral weight. The computed $\mathbf{k}$-integrated and $\mathbf{k}$-resolved spectral functions are in good agreement with photoemission spectroscopy (PES) and angular resolved PES experiments. Making use of a general classification scheme for the strength of electronic correlations we conclude that $\mathrm{BaFe}_{2} \mathrm{As}_{2}$ is a moderately correlated system.
\end{abstract}

DOI: 10.1103/PhysRevB.80.092501

The recent discovery of an entirely new class of high- $T_{c}$ superconductors based on quasi-two-dimensional FeAs-layers ${ }^{1}$ rather than on $\mathrm{CuO}$ layers has stimulated intense experimental and theoretical activity. In striking similarity with the high- $T_{c}$ cuprates, the undoped pnictides are not superconducting under ambient pressure, but exhibit an antiferromagnetic commensurate spin-density wave below $150 \mathrm{~K}^{2}{ }^{2}$ When electrons (or holes) are added to the system via doping antiferromagnetism is suppressed and superconductivity appears. Since it is generally accepted that the strong Coulomb correlations between the $\mathrm{Cu} 3 d$ electrons are responsible for the anomalous properties of cuprates, it is tempting to suggest that the same is true for the $\mathrm{Fe} 3 d$ electrons in pnictides.

Investigations of the Coulomb correlation strength in the Fe-pnictides have arrived at different conclusions. Haule, Shim, and Kotliar ${ }^{3}$ employed the LDA+DMFT approach, ${ }^{4}$ the combination of density functional theory (DFT) in the local density approximation (LDA) with the many-body dynamical mean-field theory (DMFT), ${ }^{5}$ to study $\mathrm{LaO}_{1-x} \mathrm{~F}_{x} \mathrm{Fe}$ As. Using a value of the local Coulomb interaction $U=4 \mathrm{eV}$ obtained by the random phase approximation $(\mathrm{RPA})^{6}$ for metallic iron ${ }^{7}$ [which is quite similar to that found by constrained LDA, $U=3-4 \mathrm{eV}$ (Refs. 8-10)], they concluded that $\mathrm{LaO}_{1-x} \mathrm{~F}_{x} \mathrm{FeAs}$ is a strongly correlated, bad metal, which is close to a Mott metal-insulator transition. A similar conclusion was reached by Craco et al. ${ }^{11}$ for $\mathrm{SmO}_{1-x} \mathrm{~F}_{x} \mathrm{FeAs}$. By contrast, using constrained RPA to compute $U$ in LaFeAsO Nakamura, Arita, and Imada ${ }^{12}$ obtained smaller values of $U$, in the range $U=2.2-3.3 \mathrm{eV}$. Therefore, they concluded that in $\mathrm{LaFeAsO}$ and $\mathrm{LaFePO}$, electronic correlations are moderately strong. This was affirmed by Anisimov et al. ${ }^{9}$ who performed LDA+DMFT investigations for $\mathrm{LaFeAsO}$, with $U$ taken from constrained LDA, ${ }^{13,14}$ and compared with photoemission spectroscopy (PES) data. Yet another conclusion was reached by Yang et al. ${ }^{15}$ who investigate the Fe-pnictides by $\mathrm{x}$-ray absorption spectroscopy (XAS) and resonant inelastic x-ray scattering (RIXS) and compared with results from cluster diagonalizations, multiplet and DFT calculations. They estimated the Coulomb in-
PACS number(s): 71.27.+a, 71.10.-w, 79.60.-i

teraction as $U \leq 2 \mathrm{eV}$ and inferred that the Fe-pnictides are weakly correlated systems.

These different assessments raise a general question about the classification of the strength of electronic correlations in a particular material. An overall estimate is provided by the ratio of the local Coulomb interaction $U$ and the band width $W$. For $U / W<1$, the system is considered weakly correlated, and the results of DFT approximations are sufficient to explain its electronic and magnetic properties. By contrast, if $U$ is comparable with $W$ or larger, the system is moderately or even strongly correlated, and the Coulomb interactions must be treated explicitly in electronic structure calculations. However, the single parameter $U / W$ is only a rough measure of the electronic correlations; furthermore it is not directly accessible by experiment. Here the effective mass renormalization $\mathrm{m}^{*} / \mathrm{m}$ of quasiparticles is a more useful parameter since it permits experimental tests. Nevertheless a single number cannot reliably assess the correlation strength in a real material whose properties are determined by various different aspects such as the electronic band structure, degeneracies, filling, and the Coulomb interaction parameters beyond $U$. A much more expressive quantity is the $\mathbf{k}$-resolved single-particle spectral function $A(\mathbf{k}, \omega)$, and its k-integrated variant $A(\omega)$, both of which can be measured by angular resolved photoemission spectroscopy (ARPES) and PES, respectively. They allow for an unambiguous classification of the strength of electronic correlations. Namely, if $A(\mathbf{k}, \omega)$ is well described by DFT approximations it is justified to call the system weakly correlated. If the band structure is substantially renormalized by correlation effects as expressed by an enhancement of the quasiparticle effective mass, but $A(\omega)$ does not yet show incoherent Hubbard bands, the system is moderately correlated. Only if the correlation induced transfer of spectral weight is so strong that pronounced Hubbard bands and a distinct quasiparticle peak at the Fermi energy appear, can the system be called strongly correlated (a good example is the $3 d^{1}$ system $\left.\mathrm{SrVO}_{3}\right){ }^{16}$ The spectral functions of correlated electron materials can be computed within the LDA+DMFT framework, ${ }^{4}$ which provides access to material specific single-particle spectra and higher correlation functions. 
In this Brief Report, we employ the LDA+DMFT scheme $^{4}$ to explore the importance of electronic correlation effects in $\mathrm{BaFe}_{2} \mathrm{As}_{2}$, the undoped parent compound of one of the main pnictide high- $T_{c}$ superconductor families. For this purpose we calculate the spectral functions $A(\omega)$ and $A(\mathbf{k}, \omega)$ and compare our results with the available experimental data for $\mathrm{BaFe}_{2} \mathrm{As}_{2}$. In particular, we apply the scheme described above to classify the correlation strength in this important class of Fe-pnictides.

The LDA+DMFT approach proceeds as follows: First, an effective Hamiltonian $\widetilde{h}_{\mathbf{k}}$ is constructed using converged LDA results for the system under investigation, then the many-body Hamiltonian is set up, and finally the corresponding self-consistent DMFT equations are solved. By projecting onto Wannier functions, ${ }^{17}$ we obtain an effective 16-band Hamiltonian which incorporates five $\mathrm{Fe} d$ orbitals and three As $p$ orbitals for each of the two Fe and As ions per unit cell. In the present study, we construct Wannier states for a single energy window including both $p$ and $d$ bands. Consequently, the eigenvalues of the effective Hamiltonian $\widetilde{h}_{\mathbf{k}}$ exactly correspond to the $16 \mathrm{Fe}$ and As bands from LDA. Thereby hybridization effects between As $p$ and Fe $d$ electrons are explicitly taken into account. The LDA calculations were performed with the experimentally determined crystal structure $^{18}$ using the Elk full-potential linearized augmented plane-wave (FP-LAPW) code. ${ }^{19}$ Parameters controlling the LAPW basis were kept to their default values. The calculated LDA band structure $\epsilon_{\mathrm{LDA}}(\mathbf{k})$ is found to be in good agreement with that of Fink et al. ${ }^{20}$

To account for the Coulomb interaction already present in LDA the $d d$-diagonal elements of the effective Hamiltonian $\widetilde{h}_{\mathbf{k}}$ are renormalized by a double counting correction ${ }^{4} E_{d c}$ $=U\left(n_{\mathrm{DMFT}}-\frac{1}{2}\right)$,

$$
h_{\mathbf{k}, \alpha \beta}^{d d}=\widetilde{h}_{\mathbf{k}, \alpha \beta}^{d d}-E_{d c} \delta_{\alpha \beta} .
$$

Here, $n_{\mathrm{DMFT}}$ is the total, self-consistently calculated number of $d$ electrons per Fe site obtained within the LDA+DMFT. This form of $E_{d c}$ yields reliable results for transition metal compounds, including the superconductor $\mathrm{LaFeAsO}{ }^{9}$ The $p-d$ Hamiltonian to be solved by DMFT then has the form

$$
\begin{aligned}
H= & \sum_{\mathbf{k}, \sigma}\left(h_{\mathbf{k}, \alpha \beta}^{d d} d_{\mathbf{k} \alpha \sigma}^{\dagger} d_{\mathbf{k} \beta \sigma}+h_{\mathbf{k}, \gamma \delta}^{p p} p_{\mathbf{k} \gamma \sigma}^{\dagger} p_{\mathbf{k} \delta \sigma}+h_{\mathbf{k}, \alpha \gamma}^{d p} d_{\mathbf{k} \alpha \sigma}^{\dagger} p_{\mathbf{k} \gamma \sigma}\right. \\
& \left.+h_{\mathbf{k}, \gamma \alpha}^{p d} p_{\mathbf{k} \gamma \sigma}^{\dagger} d_{\mathbf{k} \alpha \sigma}\right)+\sum_{i, \sigma, \sigma^{\prime}} U_{\alpha \beta}^{\sigma \sigma^{\prime}} n_{i \alpha \sigma}^{d} n_{i \beta \sigma^{\prime}}^{d} .
\end{aligned}
$$

Here, $d_{\mathbf{k} \alpha \sigma}$ and $p_{\mathbf{k} \gamma \sigma}$ are Fourier transforms of $d_{i \alpha \sigma}$ and $p_{i \gamma \sigma}$, which annihilate the $d$ or $p$ electron with orbital and spin indices $\alpha \sigma$ or $\gamma \sigma$ in the $i$ th unit cell, and $n_{i \alpha \sigma}^{d}$ is the corresponding occupation number operator.

The DMFT self-consistency equations were solved iteratively for imaginary Matsubara frequencies. The auxiliary impurity problem was solved by the Hirsch-Fye quantum Monte Carlo (QMC) method. ${ }^{21}$ The elements of $U_{\alpha \beta}^{\sigma \sigma^{\prime}}$ matrix are parameterized by $U$ and $J$ according to procedure described in. ${ }^{22}$ In the following we use the interaction parameters $U=3.1 \mathrm{eV}$ and $J=0.81 \mathrm{eV}$ obtained in. ${ }^{9}, 10$ Calculations were performed in the paramagnetic state at the inverse
TABLE I. Effective mass renormalization $\mathrm{m}^{*} / \mathrm{m}$ of quasiparticles in $\mathrm{BaFe}_{2} \mathrm{As}_{2}$ for different orbitals of the $d$ shell.

\begin{tabular}{lcccc}
\hline \hline Orbitals & $d_{x y}$ & $d_{y z, x z}$ & $d_{3 z^{2}-r^{2}}$ & $d_{x^{2}-y^{2}}$ \\
\hline$m^{*} / m$ & 2.06 & 2.07 & 2.05 & 1.83 \\
\hline \hline
\end{tabular}

temperature $\beta=1 / T=20 \mathrm{eV}^{-1}$. The imaginary time interval $0<\tau<\beta$ was divided into 150 slices, and $6 \times 10^{6}$ QMC sweeps were used in a self-consistency loop within the LDA+DMFT scheme. The local self-energy $\Sigma(\omega)$, which is formally an $16 \times 16$ matrix with the only nonzero elements on the diagonal of the $d d$ block, was calculated for real energies $\omega$ by analytic continuation from imaginary Matsubara frequencies using the Padé approximant. ${ }^{23}$

A quantitative measure of the electron correlation strength is also provided by the quasiparticle renormalization factor $Z=\left(1-\left.\frac{\partial \Sigma}{\partial \omega}\right|_{\omega=0}\right)^{-1}$, which leads to an effective mass enhancement $m^{*} / m=Z^{-1}$. In general, the self-energy is a matrix, leading to different effective masses for different bands. The masses can be obtained from the self-energy on the real axis, $\Sigma(\omega)$. The calculated $\mathrm{m}^{*} / \mathrm{m}$ values for every $d$-orbital are presented in Table I. The $d_{x^{2}-y^{2}}$ orbital has the smallest effective mass renormalization $\left(\mathrm{m}^{*} / \mathrm{m}=1.83\right)$. The other $d$ orbitals have a slightly larger value $\left(m^{*} / m=2.05-2.07\right)$. The overall effective mass enhancement $m^{*} / m \approx 2$ agrees well with the ARPES data for $\mathrm{BaFe}_{2} \mathrm{As}_{2} \cdot{ }^{24,25}$ It is also in good agreement with experimental results for the pnictide material $\mathrm{LaFePO}$, where de Haas-van Alphen experiments ${ }^{26}$ found a mass enhancement between 1.7 and 2.1, and ARPES ${ }^{27}$ measured a band renormalization by a factor of 2.2 compared with the LDA.

The orbitally resolved Fe $3 d$ and total As $4 p$ spectral functions computed within the LDA and LDA+DMFT, respectively, are compared in Fig. 1. The tetragonal crystal field splitting of the $\mathrm{Fe} 3 d$ states in this material is rather weak $\left(\Delta_{c f}=0.25 \mathrm{eV}\right)$. Within the LDA all five Fe $d$ orbitals form a common band in the energy range $(-2,+2) \mathrm{eV}$ relative to the Fermi level (band width $W \approx 4 \mathrm{eV}$ ). There is a significant hybridization of the $\mathrm{Fe} t_{2 g}$ orbitals with the As $p$ orbitals, leading to spectral weight in the energy interval $(-3,-2)$ eV where the As $p$ band is located. However, this is not a correlation effect. The correlations only lead to some broadening but not to substantial spectral weight transfer, i.e., there are no Hubbard bands found in the entire spectrum. Indeed, the overall shape of the LDA spectral function is hardly changed by the correlations.

In Fig. 2, the total Fe $3 d$ and As $4 p$ spectral functions are compared with photoemission spectroscopy data of de Jong et $a l .{ }^{28}$ The computed spectra reproduce the experimental features very well, including the relative positions of the $d$ and $p$ bands and the strong $d$ contribution at the top of the valence band.

We now calculate the $\mathbf{k}$-resolved spectral function

$$
A(\mathbf{k}, \omega)=-\operatorname{Im} \frac{1}{\pi} \operatorname{Tr}\left[(\omega+\mu) \hat{I}-\hat{h}_{\mathbf{k}}-\hat{\Sigma}(\omega)\right]^{-1} .
$$

Here, $\hat{h}_{\mathbf{k}}$ is the $16 \times 16$ Hamiltonian matrix on a mesh of $\mathbf{k}$-points and $\mu$ is the self-consistently determined chemical 


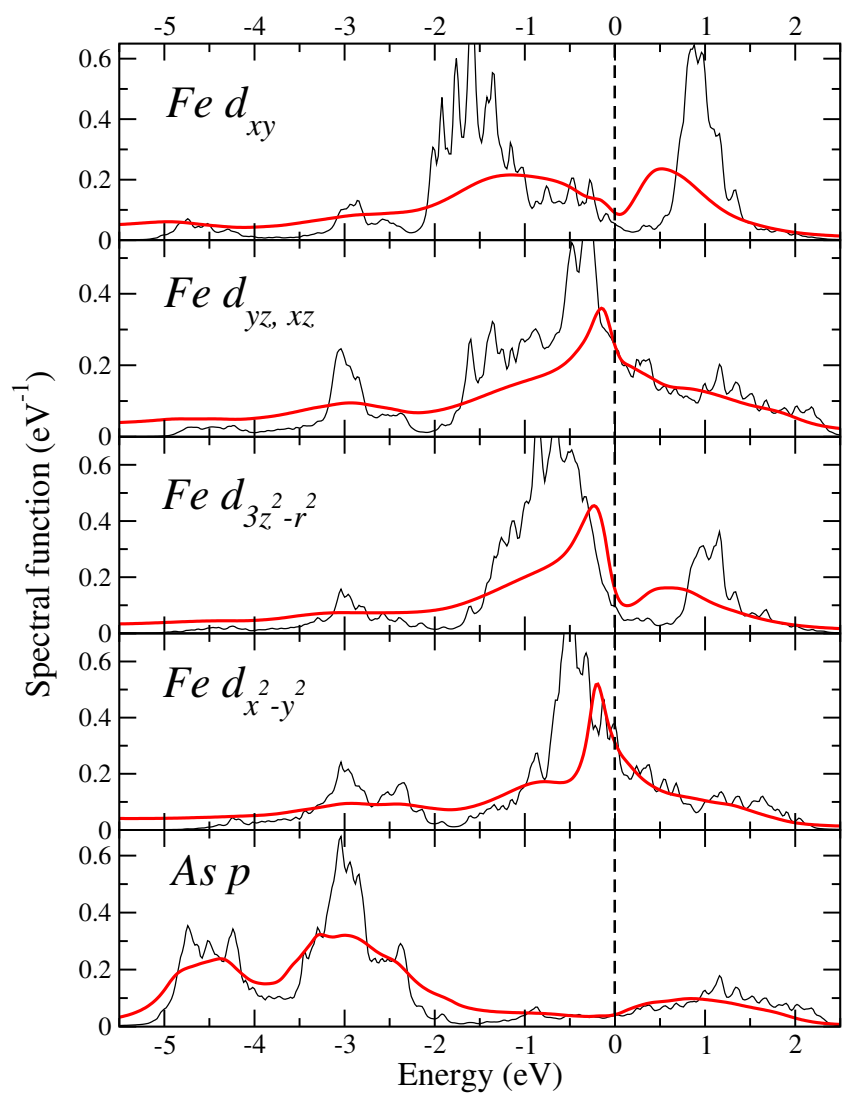

FIG. 1. (Color online) Orbitally resolved Fe $3 d$ and total As $p$ spectral functions of $\mathrm{BaFe}_{2} \mathrm{As}_{2}$ obtained within LDA+DMFT (solid lines) are compared with LDA results (shaded areas).

potential. In Fig. 3 we compare our results with ARPES data of Liu et al. ${ }^{29}$ Both theory and experiment show dispersive bands crossing the Fermi level near the $\Gamma$ and $X$ points. In addition, two weakly pronounced relatively flat bands can be seen in the region from -0.2 to $-0.3 \mathrm{eV}$ and from -0.5 to $-0.6 \mathrm{eV}$ near the $\Gamma$ point. The calculated shape and size of the electron and hole pockets centered at the $X$ and $\Gamma$ points, respectively, are in good agreement with the ARPES data.

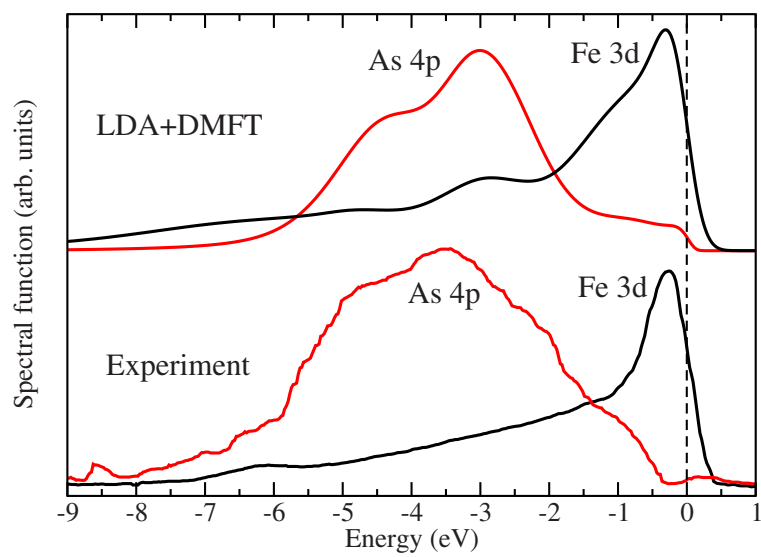

FIG. 2. (Color online) Normalized total Fe $d$ and As $p$ spectral functions of $\mathrm{BaFe}_{2} \mathrm{As}_{2}$ calculated within LDA+DMFT (upper curves) are compared with photoemission spectroscopy data of de Jong et al. (Ref. 28) (lower curves).
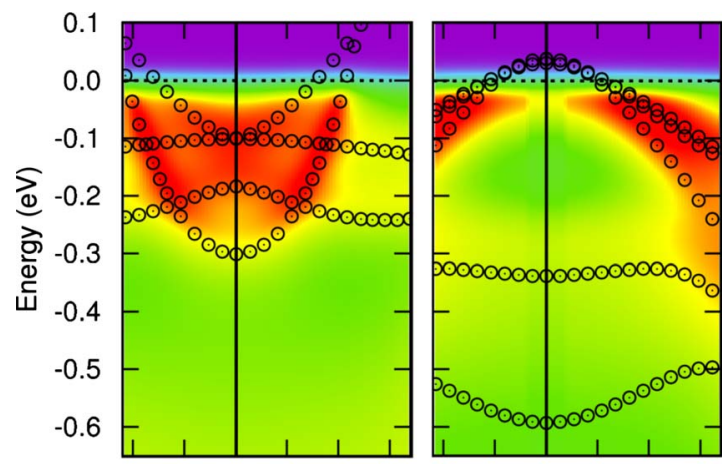

(a)

$\begin{array}{lllllllllllll}-0.2 & -0.1 & 0.0 & 0.1 & 0.2 & 0.3 & -0.2 & -0.1 & 0.0 & 0.1 & 0.2 & 0.3\end{array}$
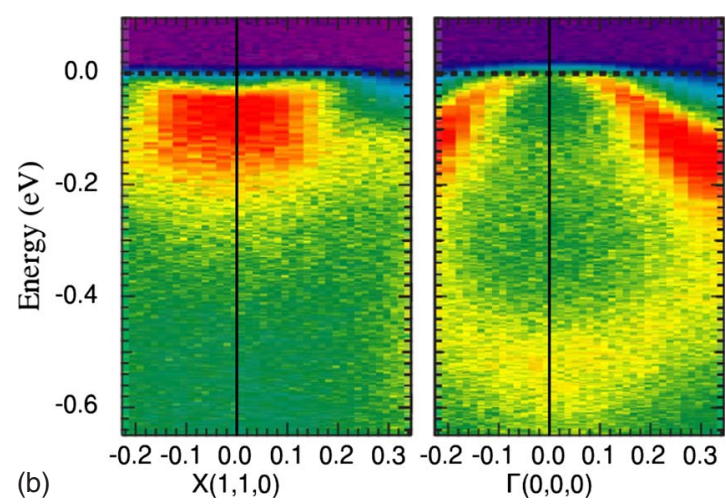

FIG. 3. (Color online) The k-resolved total spectral function $\mathrm{A}(\mathbf{k}, \omega)$ of $\mathrm{BaFe}_{2} \mathrm{As}_{2}$ near the $\Gamma$ and $X$ points in the Brillouin zone is depicted as a contour plot. Upper panel: LDA+DMFT spectral function including the renormalized band structure (circles) obtained by plotting the peak positions of the spectral function $A(\mathbf{k}, \omega)$. Lower panel: The corresponding experimental ARPES intensity map of Liu et al. (Ref. 29).

Also shown in Fig. 3 (upper panel) is the correlated band structure $\epsilon_{\mathrm{DMFT}}(\mathbf{k})$. Near the Fermi energy, i.e., in the energy range from $-0.25 \mathrm{eV}$ to zero where quasiparticles are well defined (as expressed by a linear behavior of $\operatorname{Re} \Sigma(\omega)$ ), this dispersion is very well represented by the scaling relation $\epsilon_{\mathrm{DMFT}}(\mathbf{k})=\epsilon_{\mathrm{LDA}}(\mathbf{k}) /\left(\mathrm{m}^{*} / \mathrm{m}\right)$, with $\mathrm{m}^{*} / \mathrm{m}$ as the computed mass enhancement. This shows that the band structure is substantially renormalized. Nevertheless, since there is no substantial spectral weight transfer in the spectral function, electronic correlations in $\mathrm{BaFe}_{2} \mathrm{As}_{2}$ are only moderately strong.

In conclusion, by employing the many-body dynamical mean-field theory in combination with LDA electronic band structure calculations (LDA+DMFT approach) we calculated the single-particle $\mathbf{k}$-integrated and $\mathbf{k}$-resolved spectral functions for $\mathrm{BaFe}_{2} \mathrm{As}_{2}$, a parent compound of the superconducting iron pnictide family. The correlated band structure obtained within LDA+DMFT is well described by the relation $\epsilon_{\mathrm{DMFT}}(\mathbf{k})=\epsilon_{\mathrm{LDA}}(\mathbf{k}) /\left(m^{*} / m\right)$, with an effective mass enhancement computed as $m^{*} / m \approx 2$. At the same time correlations do not lead to substantial transfer of spectral weight in the spectrum, i.e., Hubbard bands do not form. Our results are in good agreement with PES experiments of de Jong et $a l .{ }^{28}$ and ARPES data of Liu et al. ${ }^{29}$ We also discussed a general classification scheme for the electronic correlation 
strength based on the renormalization of the band structure and the transfer of spectral weight. Our results obtained for $\mathrm{BaFe}_{2} \mathrm{As}_{2}$ and their comparison with experimental data show that this material, and also other Fe-pnictides, should be classified as moderately correlated.

The authors thank Jan Kuneš for providing his DMFT(QMC) computer code used in our calculations. Support by the Russian Foundation for Basic Research under
Grant No. RFFI-07-02-00041, the Dynasty Foundation, the fund of the President of the Russian Federation for the support of scientific schools under Grant No. NSH 1941.2008.2, the Program of the Russian Academy of Science Presidium "Quantum microphysics of condensed matter" N7, the Federal Agency for Science and Innovations under Grant No. 02.740.11.0217, and the Deutsche Forschungsgemeinschaft through Sonderforschungsbereich SFB 484 is gratefully acknowledged.
${ }^{1}$ Y. Kamihara, T. Watanabe, M. Hirano, and H. Hosono, J. Am. Chem. Soc. 130, 3296 (2008); Z.-A. Ren, W. Lu, J. Yang, W. Yi, X.-L. Shen, Z.-C. Li, G.-C. Che, X.-L. Dong, L.-L. Sun, F. Zhou, and Z.-X. Zhao, Chin. Phys. Lett. 25, 2215 (2008).

${ }^{2}$ C. de la Cruz, Q. Huang, J. W. Lynn, W. Jiying Li, W. Ratcliff II, J. L. Zarestky, H. A. Mook, G. F. Chen, J. L. Luo, N. L. Wang, and Pengcheng Dai, Nature (London) 453, 899 (2008).

${ }^{3}$ K. Haule, J. H. Shim, and G. Kotliar, Phys. Rev. Lett. 100, 226402 (2008).

${ }^{4}$ K. Held, I. A. Nekrasov, G. Keller, V. Eyert, N. Blümer, A. K. McMahan, R. T. Scalettar, T. Pruschke, V. I. Anisimov, and D. Vollhardt, Phys. Status Solidi B 243, 2599 (2006); G. Kotliar, S. Y. Savrasov, K. Haule, V. S. Oudovenko, O. Parcollet, and C. A. Marianetti, Rev. Mod. Phys. 78, 865 (2006).

${ }^{5}$ W. Metzner and D. Vollhardt, Phys. Rev. Lett. 62, 324 (1989); A. Georges, G. Kotliar, W. Krauth, and M. J. Rozenberg, Rev. Mod. Phys. 68, 13 (1996); G. Kotliar and D. Vollhardt, Phys. Today 57(3), 53 (2004).

${ }^{6}$ I. V. Solovyev and M. Imada, Phys. Rev. B 71, 045103 (2005); F. Aryasetiawan, K. Karlsson, O. Jepsen, and U. Schönberger, ibid. 74, 125106 (2006).

${ }^{7}$ T. Miyake and F. Aryasetiawan, Phys. Rev. B 77, 085122 (2008).

${ }^{8}$ T. Miyake, L. Pourovskii, V. Vildosola, S. Biermann, and A. Georges, J. Phys. Soc. Jpn. 77, Supplement C, 99 (2008).

${ }^{9}$ V. I. Anisimov, Dm. M. Korotin, M. A. Korotin, A. V. Kozhevnikov, J. Kuneš, A. O. Shorikov, S. L. Skornyakov, and S. V. Streltsov, J. Phys.: Condens. Matter 21, 075602 (2009).

${ }^{10}$ V. I. Anisimov, Dm. M. Korotin, S. V. Streltsov, A. V. Kozhevnikov, J. Kuneš, A. O. Shorikov, and M. A. Korotin, JETP Lett. 88, 729 (2008); V. I. Anisimov, E. Z. Kurmaev, A. Moewes, and Y. A. Izyumov, Physica C 469, 442 (2009).

${ }^{11}$ L. Craco, M. S. Laad, S. Leoni, and H. Rosner, Phys. Rev. B 78, 134511 (2008).

${ }^{12}$ K. Nakamura, R. Arita, and M. Imada, J. Phys. Soc. Jpn. 77, 093711 (2008).

${ }^{13}$ P. H. Dederichs, S. Blügel, R. Zeller, and H. Akai, Phys. Rev. Lett. 53, 2512 (1984); O. Gunnarsson, O. K. Andersen, O. Jepsen, and J. Zaanen, Phys. Rev. B 39, 1708 (1989).

${ }^{14}$ V. I. Anisimov and O. Gunnarsson, Phys. Rev. B 43, 7570 (1991).

${ }^{15}$ W. L. Yang, A. P. Sorini, C.-C. Chen, B. Moritz, W.-S. Lee, F. Vernay, P. Olalde-Velasco, J. D. Denlinger, B. Delley, J.-H. Chu, J. G. Analytis, I. R. Fisher, Z. A. Ren, J. Yang, W. Lu, Z. X. Zhao, J. van den Brink, Z. Hussain, Z.-X. Shen, and T. P. Devereaux, Phys. Rev. B 80, 014508 (2009).

${ }^{16}$ E. Pavarini, S. Biermann, A. Poteryaev, A. I. Lichtenstein, A.
Georges, and O. K. Andersen, Phys. Rev. Lett. 92, 176403 (2004); A. Sekiyama, H. Fujiwara, S. Imada, S. Suga, H. Eisaki, S. I. Uchida, K. Takegahara, H. Harima, Y. Saitoh, I. A. Nekrasov, G. Keller, D. E. Kondakov, A. V. Kozhevnikov, Th. Pruschke, K. Held, D. Vollhardt, and V. I. Anisimov, ibid. 93, 156402 (2004); I. A. Nekrasov, K. Held, G. Keller, D. E. Kondakov, Th. Pruschke, M. Kollar, O. K. Andersen, V. I. Anisimov, and D. Vollhardt, Phys. Rev. B 73, 155112 (2006).

${ }^{17}$ V. I. Anisimov, D. E. Kondakov, A. V. Kozhevnikov, I. A. Nekrasov, Z. V. Pchelkina, J. W. Allen, S.-K. Mo, H.-D. Kim, P. Metcalf, S. Suga, A. Sekiyama, G. Keller, I. Leonov, X. Ren, and D. Vollhardt, Phys. Rev. B 71, 125119 (2005).

${ }^{18}$ Q. Huang, Y. Qiu, Wei Bao, M. A. Green, J. W. Lynn, Y. C. Gasparovic, T. Wu, G. Wu, and X. H. Chen, Phys. Rev. Lett. 101, 257003 (2008).

${ }^{19} \mathrm{http}: / /$ elk.sourceforge.net/.

${ }^{20}$ J. Fink, S. Thirupathaiah, R. Ovsyannikov, H. A. Dürr, R. Follath, Y. Huang, S. de Jong, M. S. Golden, Yu-Zhong Zhang, H. O. Jeschke, R. Valenti, C. Felser, S. Dastjani Farahani, M. Rotter, and D. Johrendt, Phys. Rev. B 79, 155118 (2009).

${ }^{21}$ J. E. Hirsch and R. M. Fye, Phys. Rev. Lett. 56, 2521 (1986); M. Jarrell and J. E. Gubernatis, Phys. Rep. 269, 133 (1996).

${ }^{22}$ A. I. Liechtenstein, V. I. Anisimov, and J. Zaanen, Phys. Rev. B 52, R5467 (1995).

${ }^{23}$ J. H. Vidberg and J. E. Serene, J. Low Temp. Phys. 29, 179 (1977).

${ }^{24}$ H. Ding, K. Nakayama, P. Richard, S. Souma, T. Sato, T. Takahashi, M. Neupane, Y.-M. Xu, Z.-H. Pan, A. V. Federov, Z. Wang, X. Dai, Z. Fang, G. F. Chen, J. L. Luo, and N. L. Wang, arXiv:0812.0534 (unpublished).

${ }^{25}$ M. Yi, D. H. Lu, J. G. Analytis, J.-H. Chu, S.-K. Mo, R.-H. He, R. G. Moore, X. J. Zhou, G. F. Chen, J. L. Luo, N. L. Wang, Z. Hussain, D. J. Singh, I. R. Fisher, and Z.-X. Shen, Phys. Rev. B 80, 024515 (2009).

${ }^{26}$ A. I. Coldea, J. D. Fletcher, A. Carrington, J. G. Analytis, A. F. Bangura, J.-H. Chu, A. S. Erickson, I. R. Fisher, N. E. Hussey, and R. D. McDonald, Phys. Rev. Lett. 101, 216402 (2008).

${ }^{27}$ D. H. Lu, M. Yi, S.-K. Mo, A. S. Erickson, J. Analytis, J.-H. Chu, D. J. Singh, Z. Hussain, T. H. Geballe, I. R. Fisher, and Z.-X. Shen, Nature (London) 455, 81 (2008).

${ }^{28}$ S. de Jong, Y. Huang, R. Huisman, F. Massee, S. Thirupathaiah, M. Gorgoi, F. Schaefers, R. Follath, J. B. Goedkoop, and M. S. Golden, Phys. Rev. B 79, 115125 (2009).

${ }^{29}$ Chang Liu, G. D. Samolyuk, Y. Lee, Ni Ni, Takeshi Kondo, A. F. Santander-Syro, S. L. Bud'ko, J. L. McChesney, E. Rotenberg, T. Valla, A. V. Fedorov, P. C. Canfield, B. N. Harmon, and A. Kaminski, Phys. Rev. Lett. 101, 177005 (2008). 